\title{
Is supraperiosteal infiltration anesthesia safe enough to prevent inferior alveolar nerve during posterior mandibular implant surgery?
}

\author{
Osman A. Etoz ${ }^{1}$, Nilay Er ${ }^{1}$, Ahmet E Demirbas ${ }^{1}$ \\ ${ }^{1}$ Department of Oral and Maxillofacial Surgery Faculty of Dentistry, Erciyes University, Kayseri, Turkey
}

Correspondence:

Erciyes Universitesi, Dis Hekimligi Fakultesi

Aglz Dis Cene Hastaliklari ve Cerrahisi Anabilim Dall, 38039, Melikgazi, Kayseri, TURKEY

osmanetoz@yahoo.com

Etoz OA, Er N, Demirbas AE. Is supraperiosteal infiltration anesthesia safe enough to prevent inferior alveolar nerve during posterior mandibular implant surgery?. Med Oral Patol Oral Cir Bucal. 2011 May 1;16 (3):e386-9.

http://www.medicinaoral.com/medoralfree01/v16i3/medoralv16i3p386.pdf

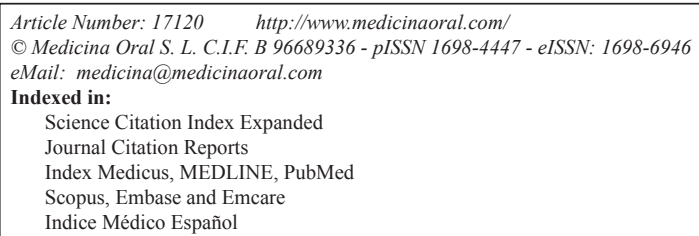

\begin{abstract}
Objectives: There is no agreement on using inferior alveolar nerve (IAN) block or supraperiosteal infiltration anesthesia during dental implant surgery in the posterior mandibular region. The aim of this study was to evaluate the effectiveness of supraperiosteal infiltration anesthesia on posterior mandibular region during dental implant surgery. Materials and Methods: In this study 52 implants were inserted under supraperiosteal infiltration anesthesia in 29 patients. After the surgery, patients were instructed to note their pain and/or painless dyscomfort on the visual analogue scale (VAS). Their pressure pain threshold (PPT) scores were evaluated by mechanical algometer. The distance between the apical end of the implants and IAN was measured by using calipers on postoperatif panoramic radiographs. Results: 50 implants to 27 patients had been able to place without pain under supraperiosteal infiltration. Implants which were placed at the mandibular second premolar and first molar region had been able to place with free of pain with supraperiosteal infiltration. There was no relationship among the distance between the apical ends of the implants and IAN with intraoperative discomfort of the patients. VAS scores during implant placement at the second premolar region were relatively higher than at the first and second molar region. Conclusion: Supraperiosteal infiltration anesthesia is a safe and effective method for posterior mandibular implant surgery. However the length of the implant should be determined carefully to avoid possible damage to IAN during implant placement under supraperiosteal infiltration anesthesia.
\end{abstract}

Key words: Supraperiosteal anesthesia; dental implant, inferior alveolar nerve, pain sensation.

\section{Introduction}

One of the most common procedures in dentistry/ oral and maxillofacial surgery is the administration of local anesthetics (1) and inferior alveolar nerve (IAN) block is a commonly used technique in dental surgery. IAN block anesthesia eliminates all somatosensory perception of the mandible, mandibular teeth, floor of the mouth, ipsilateral tongue, and all but the lateral (buccal) gingivae (2). Although this useful technique is a common procedure, the issue about its complications is a well known challenge on dental surgery. Whether IAN block anesthesia is known as one of the safest procedures in dental practice, complications or adverse reactions can still 
occur (3). Besides, in this technique there is need for additional supraperiosteal anesthesia for buccal soft tissues. The main advantage of using IAN block for is comfort of the patient and surgeon during posterior mandibular implant surgery however it has been thought that supraperiosteal infiltration may be useful for the patient to be able to sense when the IAN is in danger of being damaged (2). In the literature, whether IAN block or supraperiosteal infiltration should be used is controversial and depends on surgeon's preference. Furthermore, mandibular infiltration anesthesia has been routinely avoided in clinical practice because of its questionable efficacy. However this attitude is not based on scientific studies. Dentists are being conditioned to apply this technique since leading terms.

The aim of this study was to evaluate the effectiveness of supraperiosteal infiltration anesthesia on posterior mandibular region during dental implant surgery and to assess whether a relationship exists among the distance between the apical ends of implants inserted at the posterior mandible and IAN with intraoperative sensitivity under mandibular supraperiosteal infiltration anesthesia.

\section{Patients and Methods}

Fifty-two dental implants were inserted at the posterior mandible (posterior to the mental foramen) of total 29 patients (12 males, 17 females) under mandibular supraperiosteal infiltration anesthesia. The patients were free of any painful and neurological disorder and they were informed about the procedure and ethical approval was obtained. $2 \mathrm{ml}$ of articain including $0.010 \mathrm{mg} / \mathrm{ml}$ epinephrine was used as the local anesthetic agent. For each one of the implants total of $1 \mathrm{ml}$ anesthetic solution was deposited at the buccal and lingual sides. After 5 minutes of latent period dental implants were placed at the posterior region of the mandible. The

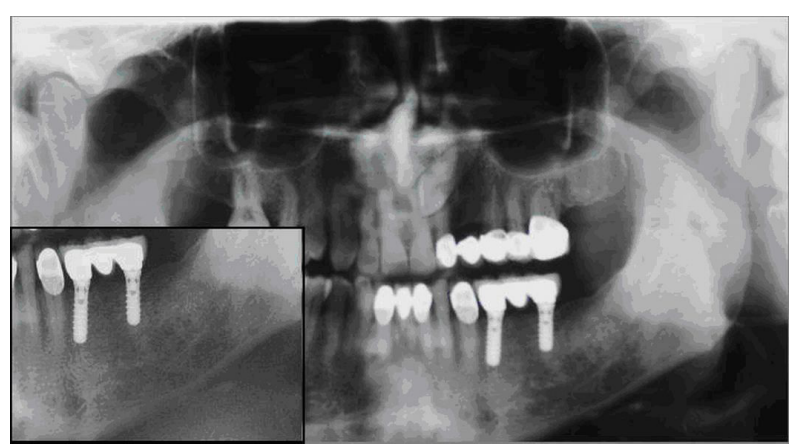

Fig. 1. Panaromic radipgraphies of the patients who underwent IAN block intaroperatively. There is no relationship between apical ends of the implants and inferior alveolar canal. patients were instructed to warn the surgeon in the case of pain sensation. After the surgery, the patients were instructed to note their intraoperative discomfort and/or pain on the visual analogue scale (VAS) of 0 to $100 \mathrm{~mm}$, which was designed as 0 being no pain and 100 being the worst pain ever experienced. Pressure pain threshold (PPT) scores of the patients were also measured with a mechanical algometer on the hypothenar region of the left hand (Wagner Instruments Greenwich, CT) in order to evaluate general pain perception of the individual (4). After surgery, standard panoramic radiographies were taken (magnification rate 1.3 ) and the distance between the apical end of the implants and IAN was measured by using calipers on postoperative panoramic radiographies. Kruskal-Wallis test and correlations were used to analyze data (SPSS 15.0, SPSS Inc, Chicago).

\section{Results}

Of 52 dental implants in 29 patients, only 2 patients underwent inferior alveolar nerve block due to pain during implant surgery. Both of the aforementioned implants were placed at the mandibular second molar region and the distance between the apical end of the implants and IANs were relatively safe (2.9 $\mathrm{mm}$ and $6.7 \mathrm{~mm}$ ) (Figs. 1,2). Remaining 27 patients (\%93.1) merely scored intraoperative painless sense on VAS and reported that they had no pain during the drilling procedure and implant placement and there was no need to use IAN block due to pain during implant surgery with the exception of two patients. Implants which were placed at the mandibular second premolar and first molar region had been able to place with free of pain with supraperiosteal infiltration. There was no association between intraoperative VAS scores of the patients and PPT values $(p>0.05)$. There was no correlation between the distance of the apical edges of implants with IAN and intraoperative VAS scores of the

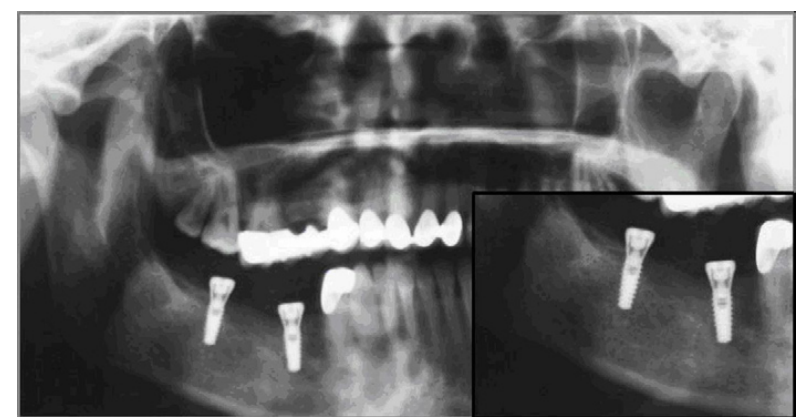

Fig. 2. Panaromic radipgraphies of the patients who underwent IAN block intaroperatively. There is no relationship between apical ends of the implants and inferior alveolar canal. 


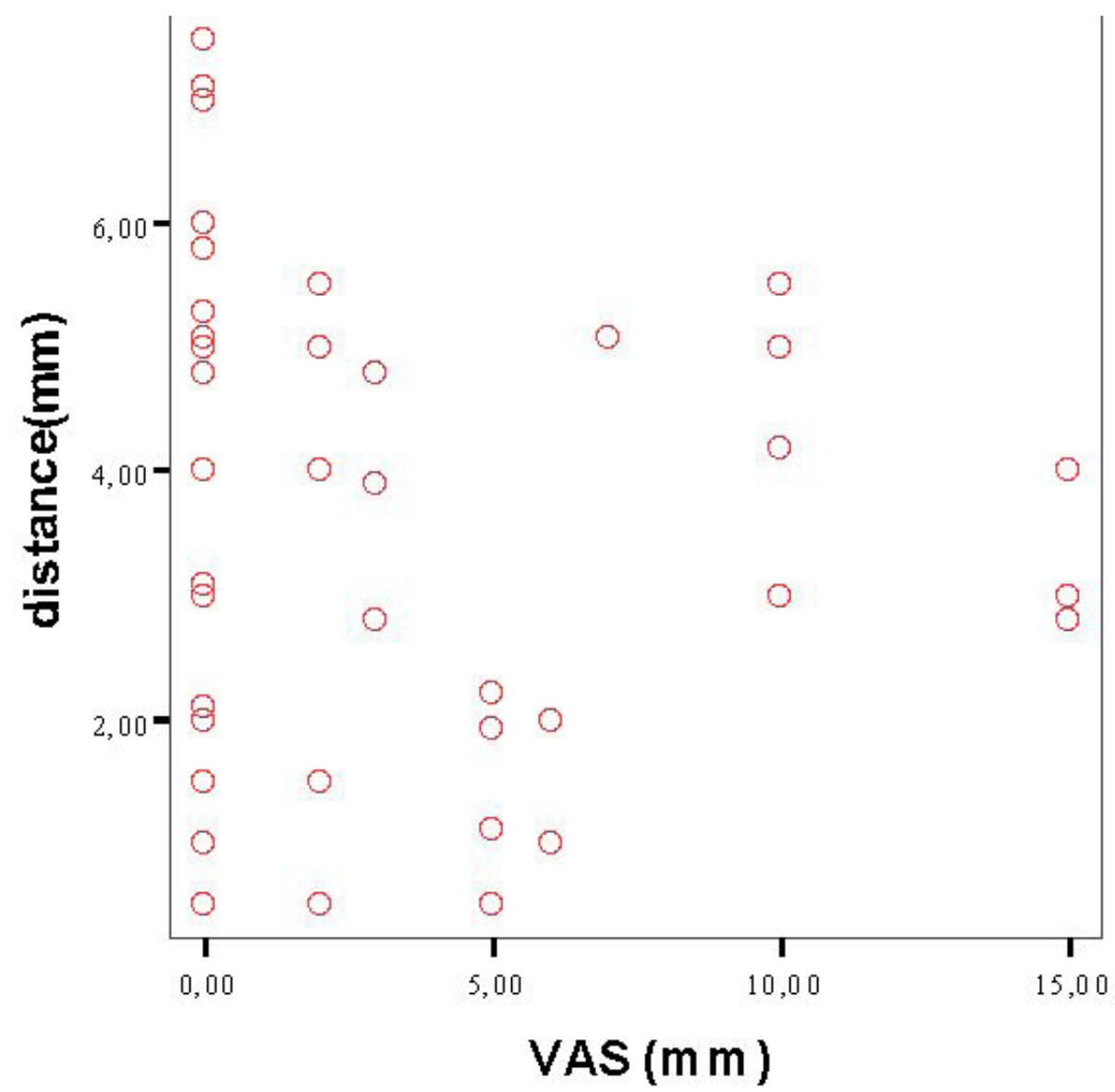

Fig. 3. Among the remaining 50 implants in 27 patients, there was no correlation between VAS scores and the distance between the apical end of the implants with IAN $(\mathrm{p}>0.05)$

Table 1. Visual analogue scale after surgery.

\begin{tabular}{|c|c|c|}
\hline & N & $\begin{array}{c}\text { Mean } \pm \text { SD of VAS } \\
\text { scores* }\end{array}$ \\
\hline SP & 10 & $6.1 \pm 5.5$ \\
FM & 30 & $2.1 \pm 3.7$ \\
\hline SM & 10 & $4.4 \pm 5.5$ \\
\hline
\end{tabular}

$* \mathrm{p}=0.048$

Abbreviations: SP, second premolar; FM, first molar; SM, second molar; SD, standard deviation; VAS, visual analogue scale; $\mathrm{N}$, number.

patients (Fig. 3) $\mathrm{p}>0.05$. Additionally, there was no association between intraoperative subjective pain scores of the patients in terms of gender and age $(\mathrm{p}>0.05)$. However, among the remaining 50 patients, VAS scores during implant placement at the second premolar region were relatively higher than at the first and second molar region and subjective pain scores were higher during the placement of dental implants at the second molar region than at the first molar region respectively (Table I) $\mathrm{p}<0.05$.

\section{Discussion}

IAN block is a useful and easy technique for management of posterior mandibular surgery. It allows a comfortable pain free environment for clinician because it assures anesthesia all of the structures that are mentioned above. Management of edentulous areas in the posterior region of the mandible with dental osseointegrated implants is usually performed under IAN block. Three major postoperative complications may occur with the use of block anesthesia of the IAN when placing mandibular implants: (i) prolonged mandibular anesthesia, during which time the patient may injure his or her tongue or lip in a variety of ways; (ii) systemic toxicity from iatrogenic, intra-arterial injection of local anesthetic solution; and, most importantly when placing mandibular implants, (iii) injury to the inferior or mental nerves, unbeknown to the patient or doctor until after the effects of the block anesthesia have subsided, sloughing of tissues, and self-inflicted soft tissue trauma $(2,3)$. In addition, although it is a rare event, some ocular complications may occur 
after IAN block. Rood, observed intermediate blindness, upper-eyelid ptosis, diplopia caused by medial strabismus, and ischemia on palatal mucosa immediately after administering anesthesia, all of which disappeared within 5-45 minutes (5). Choi et al. reported 12 cases of diploia in the English-language literature, in their systematic review (3). In the literature, it seems that ocular complications that occur immediately after IAN block, due to intrarterial injection of the local anesthetics. But even when clinicians use the utmost care, by aspirating before the injection and noting anatomic landmarks, intraarterial injections may occur during regional nerve blocks (1). Although initial aspiration may suggest that the needle tip is not in a vessel, it is quite possible for this to change during the administration of the local anesthetic solution (6). In contrast, considering all the complications and side effects of IAN block, supraperiosteal infiltration technique is easier to practice, complication rate is lesser than IAN block, and its anesthetic effect is shorter and it is much more tolerable in terms of patient's pain sufferance and postoperative comfort.

Mandibular bone is considered as too dense and too compact and because of this dense structure, it is thought that local anesthetic can not be diffused into the medullary space of mandible by supraperiosteal infiltration. However it has been found that 2449 accessory or unnamed foramina in 300 dried human mandibles (7). Madeira et al. (8) reported the presence of accessory foramina in the human mandibular symphysis region in 87.3 to $96.2 \%$ of specimens studied. In addition, Pogrel et al.(9) reported that branches of the mental nerve reenter the labial (lateral) surface of the mandible to supply lower incisors. Based on the abovementioned findings and our results, the success of supraperiosteal infiltration anesthesia at the posterior region of the mandible might be related with the possibility of diffusion of local anesthetic solution within the bony structures. However, as the compact architecture of the mandible and superficial course of the IAN increase through the second molar region, patients may express higher sensitivity during implant placement which might be a possible reason of higher VAS scores at the second molar region.

Many surgeons recommend that, to avoid mandibular nerve injury, a 2-mm radiographic space above the mandibular canal should remain after implant placement (10). In our study, results showed that there is no correlation between the distance between apical edges of the implants and inferior alveolar canal with patient sensation. Our results are consistent with thoughts of Flanagan, in which there is no recommended safe distance to avoid possible nerve damage (10).

\section{Conclusion}

Considering potential complications of IAN block, infiltration anesthesia can be used for management of posterior mandibular implant surgery especially at first molar region. Implants which were placed at the mandibular second premolar and first molar region had been able to place with free of pain with supraperiosteal infiltration. VAS scores during implant placement at the second premolar region were relatively higher than at the first and second molar region. According to the results of the present study, mandibular supraperiosteal infiltration anesthesia has a success rate of more than $90 \%$ and could be used safely for posterior mandibular implant surgery. Although supraperiosteal infiltration has been thought to permit the patient to inform the doctor if his or her surgical procedures were stimulating branches of the inferior alveolar plexus (2), preoperative size of the implant should be determined carefully in order to avoid possible damage to IAN under supraperiosteal infiltration as there was no relationship between the sensation of the patient and distance between the apical end of the implant with IAN.

\section{References}

References with links to Crossref - DOI

1. Uckan S, Cilasun U, Erkman O. Rare ocular and cutaneous complication of inferior alveolar nerve block. J Oral Maxillofac Surg. 2006;64:719-21.

2. Heller AA, Shankland WE 2nd. Alternative to the inferior alveolar nerve block anesthesia when placing mandibular dental implants posterior to the mental foramen. J Oral Implantol. 2001;27:127-33.

3. Choi EH, Seo JY, Jung BY, Park W. Diplopia after inferior alveolar nerve block anesthesia: report of 2 cases and literature review. Oral Surg Oral Med Oral Pathol Oral Radiol Endod. 2009;107:e21-4.

4. Etöz OA, Ataoğlu H. Evaluation of pain perception in patients with temporomandibular disorders. J Oral Maxillofac Surg. 2007;65:2475-8.

5. Rood JP. Ocular complication of inferior dental nerve block. A case report. Br Dent J. 1972;132:23-4.

6. Paul R, Anand R, Wray P, D'sa S, Brennan PA. An unusual complication of an inferior dental nerve block: a case report. Br Dent J. 2009;206:9-10.

7. Sutton RN. The practical significance of mandibular accessory foramina. Aust Dent J. 1974;19:167-73.

8. Madeira MC, Percinoto C, das Graças M Silva M. Clinical significance of supplementary innervation of the lower incisor teeth: a dissection study of the mylohyoid nerve. Oral Surg Oral Med Oral Pathol. 1978;46:608-14.

9. Pogrel MA, Smith R, Ahani R. Innervation of the mandibular incisors by the mental nerve. J Oral Maxillofac Surg. 1997;55:961-3.

10. Flanagan D. Delayed onset of altered sensation following dental implant placement and mental block local anesthesia: a case report. Implant Dent. 2002;11:324-30. 